\title{
Tendências recentes dos óbitos fetais por malformações congênitas: um estudo descritivo
}

\author{
Recent trends in fetal death due to congenital malformations: a \\ descriptive study
}

\section{Tendencias recientes de los óbitos de fetos por malformaciones congénitas: un estudio descriptivo}

\author{
Vanalda Costa Silva1,a \\ vanaldacosta@gmail.com | https://orcid.org/0000-0002-9495-7158
}

Rômulo Cesar Rezzo Pires ${ }^{2, b}$

romulo.pires@yahoo.com.br | https://orcid.org/o000-0003-0967-3351

Andréa Martins Cantanhede ${ }^{3, c}$

andrea.cantanhede@ufma.br | https://orcid.org/0000-0002-3290-0236

\footnotetext{
${ }^{1}$ Escola Técnica do SUS. São Luís, MA, Brasil.

${ }^{2}$ Faculdade do Maranhão, Núcleo Interdisciplinar de Pesquisa e Extensão em Enfermagem. São Luís, MA, Brasil.

${ }^{3}$ Universidade Federal do Maranhão. Chapadinha, MA, Brasil.

a Especialização em Estratégia Saúde da Família pelo Instituto Florence de Ensino Superior.

b Mestrado em Saúde e Ambiente pela Universidade Federal do Maranhão.

'Doutorado em Genética, Conservação e Biologia Evolutiva pelo Instituto de Pesquisas da Amazônia.
}

\section{Resumo}

Este artigo se baseia em um estudo de série temporal sobre os óbitos fetais por malformações congênitas no estado do Maranhão relativo ao período de 2006 a 2016. Foram construídos indicadores epidemiológicos para estimar o risco de morte fetal e sua tendência ao longo da série estudada. Os dados são provenientes do Departamento de Informática do SUS e sua análise realizada por modelos de regressão linear. Foram registrados 17.843 óbitos fetais no período abordado pelo estudo, 528 dos quais decorrentes de malformações congênitas (2,96\%). Observou-se uma tendência significativa de aumento do coeficiente de mortalidade fetal geral, correspondente a $6,99 \%(\beta 1=0,17 ; \mathrm{p}=0,004)$ e do específico por malformações congênitas, equivalente a $5,13 \%(\beta 1=0,01 ; p=0,04)$. Os resultados deste estudo corroboram a tendência histórica dos serviços de saúde negligenciarem os óbitos fetais. É importante destacar que parte destes óbitos são preveníveis e potencialmente evitáveis. Desse modo, a implementação dos comitês de investigação de óbitos fetais e infantis e a sua vigilância adequada poderiam melhorar a assistência prestada tanto no pré-natal quanto no parto.

Palavras-chave: Óbito fetal; Sistema de informação; Malformação congênita; Mortalidade fetal; Epidemiologia. 


\begin{abstract}
This article bases on a time series study about fetal deaths due to congenital malformations in the state of Maranhão, Brazil, occurred from 2006 to 2016. Epidemiological indicators were constructed to estimate the risk of fetal death and its trend throughout the series studied. The data were obtained in the Department of Informatics of SUS and analyzed by linear regression models. There were 17,843 fetal deaths during the analysed period, from which 528 were a direct result of congenital malformations (2.96\%). A significant tendency towards an increase in the coefficient of general fetal mortality corresponding to $6.99 \%\left(\beta_{1}=0.17\right.$; $\mathrm{p}=0.004)$ and in the coefficient of specific fetal mortality due to congenital malformations equivalent to $5.13 \%\left(\beta_{1}=0.01 ; \mathrm{p}=0.04\right)$ were observed. The end results of this study corroborate the historical trend toward negligence in Brazilian health centres with regard to fetal deaths. It is important to remark that some of these deaths can be presumed and potentially preventable. Thus, the implementation of the fetal and infant death investigation committees and their adequate surveillance could improve care during prenatal and delivery.
\end{abstract}

Keywords: Fetal death; Information system; Congenital malformation; Fetal mortality; Epidemiology.

\title{
Resumen
}

Este artículo se basa en un estudio de serie temporal acerca de muertes de fetos por malformaciones congénitas en el estado de Maranhão, Brasil, concerniente al periodo de 2006 a 2016. Se construyeron indicadores epidemiológicos para estimar el riesgo de la muerte fetal y su tendencia a lo largo de la serie estudiada. Los datos son provenientes del Departamento de Informática del SUS y fueron analizados por modelos de regresión lineal. Se registraron 17.843 muertes de fetos en el período estudiado, de los cuales 528 fueron resultado de malformaciones congénitas $(2,96 \%)$. Se observó una tendencia significativa al aumento del coeficiente de mortalidad fetal general correspondiente a $6.99 \%(\beta 1=0,17 ; \mathrm{p}=0,004)$ y del específico, por malformaciones congénitas, equivalente a 5,13\% $(\beta 1=0,01 ; \mathrm{p}=0,04)$. Los resultados del estudio corroboran la tendencia histórica a la negligencia de los centros de salud brasileños con respecto a las muertes de los fetos. Por su importancia debemos destacar que parte de esas muertes son presumibles y pueden ser evitadas. De ese modo, la implementación de los comités de investigación de muertes de fetos y infantiles y su vigilancia adecuada podrían mejorar la asistencia prestada en el prenatal y en el parto.

Palabras clave: Muerte fetal; Sistema de información; Malformaciones congénitas; Mortalidad fetal; Epidemiología.

Contribuição dos autores:

Concepção e desenho do estudo: Rômulo Cesar Rezzo Pires, Vanalda Costa Silva, Andréa Martins Cantanhede.

Aquisição, análise ou interpretação dos dados: Rômulo Cesar Rezzo Pires, Vanalda Costa Silva, Andréa Martins Cantanhede.

Redação do manuscrito: Rômulo Cesar Rezzo Pires.

Revisão crítica do conteúdo intelectual: Rômulo Cesar Rezzo Pires, Vanalda Costa Silva, Andréa Martins Cantanhede.

Declaração de conflito de interesses: não há.

Fontes de financiamento: não houve.

Considerações éticas: não há.

Agradecimentos/Contribuições adicionais: não há.

Histórico do artigo: submetido: 4 jun. 2018 | aceito: 15 out. 2019 | publicado: 20 dez. 2019.

Apresentação anterior: não houve.

Licença CC BY-NC atribuição não comercial. Com essa licença é permitido acessar, baixar (download), copiar, imprimir, compartilhar, reutilizar e distribuir os artigos, desde que para uso não comercial e com a citação da fonte, conferindo os devidos créditos de autoria e menção à Reciis. Nesses casos, nenhuma permissão é necessária por parte dos autores ou dos editores. 


\section{Introdução}

Óbito fetal é definido como a morte do produto da gestação antes da expulsão ou de sua extração completa do corpo materno, independentemente da duração da gravidez. O diagnóstico é confirmado se o feto não respirar nem der nenhum sinal de vida como batimentos cardíacos, pulsação do cordão umbilical ou movimentos efetivos dos músculos de contração voluntária ${ }^{1}$.

Para fins epidemiológicos, os óbitos fetais são monitorados por meio do coeficiente de mortalidade fetal (CMF), expresso pela razão entre o número de óbitos fetais e o número de nascidos (vivos e mortos), multiplicado por mil ${ }^{1}$. Este indicador é bastante sensível às condições de saúde de uma população, refletindo tanto o estado global de saúde materna como a qualidade e a acessibilidade aos cuidados primários de saúde disponíveis para gestantes e, ainda, a qualidade da assistência intraparto².

Estima-se a ocorrência mundial de 2,6 milhões de mortes fetais por ano, sendo que cerca de $98 \%$ destas mortes ocorrem em países pobres ou em desenvolvimento ${ }^{3}$. Um estudo de série histórica sobre óbito fetal relativo ao período de 1996 a 2015 mostrou tendência estacionária na taxa de mortalidade fetal (TMF) a partir do ano 2000 no Brasil, como um todo, e em cada uma de suas macrorregiões. No entanto, a taxa de mortalidade fetal do país passou de 8,19, em 1996, para 9,50 por mil nascimentos em 2015. Houve tendência crescente dos óbitos fetais cuja causa básica foram as malformações congênitas, deformidades e anomalias cromossômicas no Brasil e em todas as suas macrorregiões ${ }^{4}$.

A Organização Mundial de Saúde (OMS) e o Fundo Internacional de Emergência das Nações Unidas para as Crianças (Unicef, na sigla em inglês) estabeleceram, em 2014, uma meta a ser alcançada em 2030, a de reduzir a mortalidade fetal para 12 ou menos natimortos em cada mil nascimentos ${ }^{3,5}$. Desse modo, algumas unidades federativas do Brasil ainda estão distantes desta realidade.

Estudos epidemiológicos sobre mortalidade fetal no Brasil são escassos e as análises disponíveis na literatura e estatísticas muito restritas, refletindo a baixa visibilidade e interesse das autoridades públicas diante deste agravo ${ }^{1}$. Entretanto, o número de óbitos fetais reflete o insucesso do processo gestacional, seja para mulher ou para o profissional de saúde responsável por sua vigilância ${ }^{6}$.

A etiologia do óbito fetal revela tratar-se de um problema multifatorial, e inúmeros fatores têm sido associados a esse desfecho, embora entre $40 \%$ e 50\% dos casos sejam idiopáticos ${ }^{8}$. Além disso, entre $25 \%$ e $62 \%$ destas mortes são consideradas preveníveis, e poderiam ser reduzidas com a melhoria dos cuidados durante o pré-natal, o pronto conhecimento das complicações intraparto e o rápido encaminhamento da mulher ao hospital7.

Entre os fatores associados ao óbito fetal, destacam-se determinantes distais (socioeconômicos), intermediários (assistência) e proximais (biológicos) ${ }^{8,9}$. Merece destaque a ocorrência de malformações congênitas como fator fortemente associado ao óbito fetal. Uma revisão sistemática da literatura brasileira sobre os óbitos fetais no Brasil, publicada no período de 2003 a 2013, mostrou que a presença de malformações congênitas aumenta a chance de ocorrência de óbitos fetais medida pela Odds Ratio (OR) (OR=9,7 em Caxias do Sul, RS; OR=7,5 em Recife, PE e OR=4,17 em 14 distritos da cidade de São Paulo, SP $)^{10}$.

Com base nesse cenário de negligência e de poucos investimentos para evitar os óbitos fetais, foi publicada em 2010 a Portaria Ministerial $n^{\circ} 72$ de 11 de janeiro, que estabeleceu a vigilância e investigação obrigatórias dos óbitos infantis e fetais no âmbito do Sistema Único de Saúde (SUS), bem como a identificação de fatores de risco que possam subsidiar estratégias de prevenção de casos novos ${ }^{11}$.

No Brasil, a vigilância dos óbitos fetais compreende as seguintes etapas: i) identificação do óbito; ii) aplicação dos critérios de inclusão/exclusão; iii) entrevista domiciliar; iv) levantamento de dados dos serviços de saúde (prontuários de unidades básicas de saúde, dos serviços de urgência, de ambulatório de especialidades; prontuários hospitalares; laudos de necropsia/anatomopatológico); v) resumo, discussão 
e conclusão sobre o caso; vi) análise de evitabilidade e vii) identificação das medidas de prevenção/ intervenção necessárias ${ }^{1}$. No estado do Maranhão, a vigilância dos óbitos fetais segue estas diretrizes, sendo que os comitês de prevenção reúnem-se mensalmente e avaliam a possibilidade de evitar os óbitos fetais utilizando os critérios de evitabilidade de acordo com a lista de mortes evitáveis por intervenções do SUS, com base nas declarações de óbito e fichas de investigação de óbito infantil e fetal.

A equipe de vigilância e/ou o comitê de investigação do óbito fetal deve analisar cada óbito sob o enfoque da evitabilidade, tomando como referência os possíveis problemas ocorridos nos diversos momentos da assistência de saúde e propiciando a análise da avaliação de evitabilidade do óbito por ação oportuna e resolutiva do setor saúde, incorporando o conceito de evento-sentinela da atenção de saúde ${ }^{1}$.

Apesar do trabalho desenvolvido pelos mencionados comitês, o estado do Maranhão ainda possui indicadores socioeconômicos ${ }^{12}$ e de assistência materno-infantil baixos além de uma ausência de estudos sobre mortalidade fetal. Desse modo, visando contribuir para a redução do problema, este estudo investigou os fatores associados aos óbitos fetais por malformações congênitas no Maranhão entre os anos de 2006 e 2016.

\section{Método}

Realizou-se um estudo ecológico de séries temporais retrospectivo com as notificações de óbitos fetais no estado do Maranhão ocorridos no período de 2006 a 2016.

Em 2015, o estado tinha população estimada em 6.904.241 habitantes ${ }^{13,14}$; seu Índice de Desenvolvimento Humano Municipal (IDHM), em 2010, era de 0,639, número considerado médio. Em 2016, a renda per capita no estado é de $\mathrm{R} \$ 461,00$ com $63,58 \%$ da população sujeita à pobreza ${ }^{15}$. O índice de analfabetismo ainda é um dos principais entraves ao desenvolvimento do estado, que, até 2014, tinha 20,88\% da população maior de 15 anos analfabeta ${ }^{16}$. Quanto ao saneamento básico, $82,12 \%$ da população do estado é servida por abastecimento de água encanada e 79,08\% dessa população é atendida pela coleta de resíduos sólidos urbanos, de acordo com dados de $2015^{17}$.

Considerou-se como variável dependente a ocorrência de óbitos fetais específicos por malformação congênita (MC), expressa como coeficiente de mortalidade fetal específico por malformação congênita (MMFmc).

Óbitos fetais são definidos como aqueles ocorridos a partir da $22^{\mathrm{a}}$ semana completa de gestação, ou 154 dias; ou fetos com peso igual ou superior a $500 g$; ou estatura a partir de $25 \mathrm{~cm}$ por mil nascidos totais, na população residente, no ano considerado'.

Considerou-se malformação congênita a presença de qualquer alteração estrutural no nascimento, diagnosticada através de exame clínico e/ou por exames complementares, como radiografia, ultrassonografia e ecocardiografia ${ }^{18}$.

As malformações congênitas incluem o grupo 1 da Classificação de Wigglesworth Expandida dos óbitos fetais e infantis no Brasil ${ }^{19}$.

Para a construção de indicadores, nos numeradores foram incluídos tanto óbitos fetais totais (Capítulo XV da CID-10) quanto aqueles por malformação (Capítulo XVII da CID-10 - Qoo-Q99, Malformações congênitas, deformidades e anomalias cromossômicas).

Nos denominadores, utilizou-se a população sob risco, ou seja, aquela da qual se originaram os casos. Os óbitos foram fornecidos pelo Sistema de Informações de Mortalidade (SIM) ${ }^{20}$. Os óbitos fetais e nascidos vivos com malformação foram obtidos a partir do Sistema de Informações de Nascidos Vivos (Sinasc) ${ }^{21}$. Os coeficientes foram calculados tendo como base cada mil nascidos.

Os indicadores utilizados neste estudo foram sumarizados no Quadro 1. 
Quadro 1 - Indicadores epidemiológicos de mortalidade fetal no estado do Maranhão, 2006-2016

\begin{tabular}{|l|l|l|l|}
\hline Indicador & Descrição & Cálculo & Fonte(s) \\
\hline $\begin{array}{l}\text { Óbitos fetais proporcionais } \\
\text { por MC (\%) }\end{array}$ & $\begin{array}{l}\text { Expressa a proporção de óbitos } \\
\text { por MC em relação ao total de } \\
\text { óbitos fetais }\end{array}$ & $\begin{array}{l}\text { Número de óbitos fetais por MC/ } \\
\text { Total de óbitos fetais X 100 }\end{array}$ & SIM \\
\hline $\begin{array}{l}\text { Coeficiente de mortalidade } \\
\text { fetal (\%o) }\end{array}$ & $\begin{array}{l}\text { Expressa a relação entre o } \\
\text { número de óbitos fetais e o } \\
\text { total de nascimentos }\end{array}$ & $\begin{array}{l}\text { Número de óbitos fetais/total de } \\
\text { nascidos X } 1.000\end{array}$ & SIM/Sinasc \\
\hline $\begin{array}{l}\text { Coeficientedemortalidadefetal } \\
\text { específico por malformações } \\
\text { congênitas (\%o) }\end{array}$ & $\begin{array}{l}\text { Expressa a relação entre o } \\
\text { número de óbitos fetais por } \\
\text { MC e o total de nascimentos }\end{array}$ & $\begin{array}{l}\text { Número de óbitos fetais por MC/ de nascidos X 1.000 } \\
\text { total }\end{array}$ & SIM/Sinasc \\
\hline
\end{tabular}

Fonte: Os autores (2018).

Com o objetivo de diminuir o viés de informação por subnotificação, utilizou-se a recomendação do Ministério da Saúde ${ }^{1}$ que preconiza que se some, tanto ao numerador como ao denominador, o número de óbitos fetais com idade gestacional ignorada ou não preenchida.

A análise da tendência dos coeficientes foi realizada por meio de modelos de regressão linear simples, após testados os pressupostos deste tipo de modelo.

O modelo de regressão linear foi utilizado após a técnica de ajustamento de curvas, que considera que o melhor modelo é aquele com melhor coeficiente de determinação em função da localização dos dados no gráfico de dispersão.

A análise de pressupostos para aplicação da regressão linear indicou que os valores da média e da mediana para óbitos fetais proporcionais por MC (2,96 e 3,12, respectivamente), para o coeficiente de mortalidade fetal geral (13,32 e 13,29, respectivamente) e para o coeficiente de mortalidade fetal específico por malformações congênitas (o,41 e 0,39, respectivamente) foram relativamente próximos. Os coeficientes de variação foram de 19,13\%; 5,44\% e 21,02\%, respectivamente, indicando distribuição homogênea da variável (baixa e média dispersão). A normalidade também foi atestada pelo teste de Shapiro-Wilk, apresentando valores de p variando entre 0,47025 e 0,47032.

A modelagem estatística considerou cada taxa média dos desfechos em estudo como variável dependente e o tempo cronológico (de 2006 a 2016) sob análise como variável independente. Neste sentido, foram ajustados modelos de regressão linear simples para cada série temporal, utilizando-se como valor central da série $o$ ano de 2011. Nesses modelos, $Y$ corresponde à taxa de mortalidade, $\beta_{\mathrm{o}}$ ao coeficiente anual médio, $\beta_{1}$ ao coeficiente de efeito linear (velocidade) e $\mathrm{X}$ ao ano. A tendência foi considerada significativa quando o modelo obteve $\mathrm{p}<0,05$. A qualidade do ajuste foi medida pelo coeficiente de determinação $\left(\mathrm{R}^{2}\right)^{22-25}$. As análises estatísticas foram realizadas no programa BioEstat $5 \cdot 3^{26}$.

Com o intuito de estimar o aumento ou a redução do número de mortes nos anos investigados, foi calculada a variação percentual relativa, contabilizada a partir da diferença entre o valor do ano final do período de tempo estudado e o valor observado no ano de início, dividida pelo valor do ano de início. Em adição, utilizou-se a classificação das causas evitáveis pela CID-10 ${ }^{1}$.

O estudo utilizou dados secundários de domínio público e irrestrito contidos na plataforma DATASUS. Diante disso, não foi apreciado por Comitê de Ética em Pesquisa com Seres Humanos (CEP). 


\section{Resultados}

No período de estudo, o número de nascidos vivos foi 1.323.856, o de óbitos fetais chegou a 17.843 e o de óbitos fetais por malformação congênita ficou em 528, o que representou 2,96\% da casuística dos óbitos fetais (Tabela 1). Após classificação do óbito fetal por MC em função da categoria CID-10, a proporção de evitabilidade do óbito foi da ordem de $58,14 \%$.

O ano de 2015 foi o que apresentou o maior número de notificações de estatísticas vitais (nascimentos totais e óbitos fetais), bem como os maiores valores dos indicadores de mortalidade selecionados para o estudo (Tabela 1).

No período estudado, houve diminuição discreta dos óbitos fetais $(-7,35 \%)$, dos óbitos fetais por malformações congênitas (-9,80\%), dos óbitos fetais proporcionais por malformações congênitas $(-2,50 \%)$ e do número de nascimentos totais, ou seja, nativivos e natimortos (-13,38\%). Por outro lado, o coeficiente de mortalidade fetal geral teve aumento discreto $(+6,99 \%)$, bem como o coeficiente de mortalidade fetal específico por malformações congênitas $(+5,13 \%)$ (Tabela 1$)$.

Tabela 1 - Estatísticas vitais e indicadores epidemiológicos de mortalidade fetal no estado do Maranhão, 2006-2016

\begin{tabular}{lccccccc}
\hline Ano & $\begin{array}{c}\text { Óbitos } \\
\text { fetais }\end{array}$ & $\begin{array}{c}\text { Óbitos } \\
\text { fetais } \\
\text { por MC }\end{array}$ & $\begin{array}{c}\text { Óbitos } \\
\text { fetais } \\
\text { por MC } \\
\text { (\%) }\end{array}$ & $\begin{array}{c}\text { Óbitos } \\
\text { fetais }+ \\
\text { nascidos } \\
\text { vivos }\end{array}$ & $\begin{array}{c}\text { Nascidos } \\
\text { vivos }\end{array}$ & $\begin{array}{c}\text { Coeficiente } \\
\text { de } \\
\text { mortalidade } \\
\text { fetal (\%o) }\end{array}$ & $\begin{array}{c}\text { Coeficiente de } \\
\text { mortalidade fetal } \\
\text { por malformações } \\
\text { congênitas (\%o) }\end{array}$ \\
\hline 2006 & 1.591 & 51 & 3,20 & 129315 & 127.724 & 12,30 & 0,39 \\
2007 & 1.634 & 35 & 2,14 & 128941 & 127.307 & 12,67 & 0,27 \\
2008 & 1.587 & 47 & 2,96 & 129889 & 128.302 & 12,22 & 0,36 \\
2009 & 1.666 & 55 & 3,30 & 125301 & 123.635 & 13,29 & 0,44 \\
2010 & 1.663 & 29 & 1,74 & 121229 & 119.566 & 13,72 & 0,24 \\
2011 & 1.627 & 52 & 3,20 & 122736 & 121.109 & 13,26 & 0,42 \\
2012 & 1.565 & 53 & 3,39 & 117604 & 116.039 & 13,31 & 0,45 \\
2013 & 1.641 & 46 & 2,80 & 116641 & 115.000 & 14,07 & 0,39 \\
2014 & 1.673 & 50 & 2,99 & 118744 & 117.071 & 14,09 & 0,42 \\
2015 & 1.722 & 64 & 3,72 & 119286 & 117.564 & 14,43 & 0,54 \\
2016 & 1.474 & 46 & 3,12 & 112013 & 110.539 & 13,16 & 0,41 \\
\hline Totala/Geral ${ }^{\mathrm{b}}$ & $17.843^{\mathrm{a}}$ & $528^{\mathrm{a}}$ & $2,96^{\mathrm{b}}$ & $1.341 .699^{\mathrm{a}}$ & $1.323 .856^{\mathrm{a}}$ & $13,29^{\mathrm{b}}$ & $0,40^{\mathrm{b}}$ \\
\hline
\end{tabular}

Fonte: Os autores (2018).

Com relação à análise da evolução dos indicadores selecionados no período de 2006 a 2016, observouse uma tendência crescente e estatisticamente significativa dos coeficientes de mortalidade fetais gerais $\left(\beta_{0}=13,29\right.$ e $\left.\beta_{1}=0,17\right)$ e dos específicos por malformações congênitas $\left(\beta_{0}=0,41, \beta_{1}=0,01\right)$. Por outro lado, os óbitos fetais proporcionais por malformação congênita não apresentaram tendência significativa no período (Tabela 2 e Figura 1). 
Tabela 2 - Parâmetros da regressão linear para os indicadores de mortalidade fetal no estado do Maranhão, 2006-2016

\begin{tabular}{lcccccc}
\hline Indicador & $\mathbf{r}$ & $\boldsymbol{\beta}_{\mathbf{0}}$ & $\boldsymbol{\beta}_{\mathbf{1}}$ & pvalue & $\mathbf{R}^{\mathbf{2}}$ & Equação \\
$\begin{array}{l}\text { Óbitos fetais proporcionais } \\
\text { por malformações congênitas }\end{array}$ & 0,22 & 2,95 & 0,04 & 0,50 & $05,48 \%$ & $\mathrm{y}=2,95+0,04(\mathrm{x}-2011)$ \\
$\begin{array}{l}\text { Coeficiente de mortalidade } \\
\text { fetal geral }\end{array}$ & 0,78 & 13,29 & 0,17 & 0,004 & $57,84 \%$ & $\mathrm{y}=13,29+0,17(\mathrm{x}-2011)$ \\
$\begin{array}{l}\text { Coeficiente de mortalidade } \\
\text { fetal específico por } \\
\text { malformações congênitas }\end{array}$ & 0,60 & 0,41 & 0,01 & 0,04 & $19,61 \%$ & $\mathrm{y}=0,41+0,01(\mathrm{x}-2011)$ \\
\hline
\end{tabular}

Fonte: Os autores (2018)

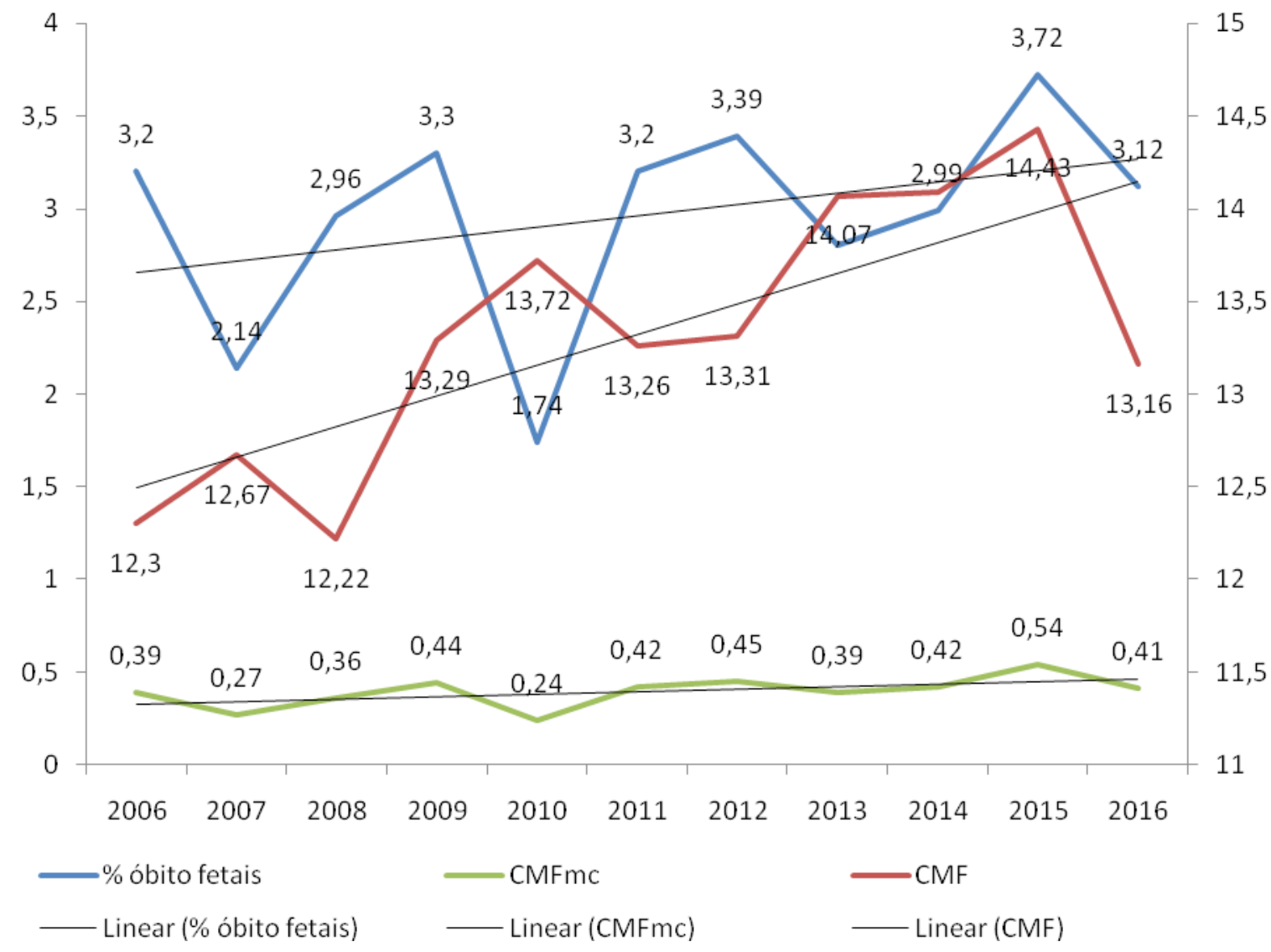

Figura 1 - Tendência dos indicadores de mortalidade fetal no estado do Maranhão, 2006-2016.

Legenda: CFM (Coeficiente de mortalidade fetal \%o nascidos); CFMmc (Coeficiente de Mortalidade Fetal por malformações congênitas \%o nascidos); e \% Óbitos Fetais (proporção de óbitos fetais por malformações congênitas).

Fonte: Os autores (2018)

Os coeficientes de correlação para os indicadores de mortalidade fetais gerais $(r=0,78)$ e específicos por malformações congênitas $(\mathrm{r}=\mathrm{0}, 60)$ apresentaram correlações positivas significativas fortes quando se considerou, como variável dependente, o indicador de mortalidade e, como variável explicativa, o tempo cronológico. Já a proporção de óbitos fetais por malformação congênita não se correlacionou com a variável explicativa (Tabela 2).

As causas de óbito fetal relacionadas às malformações congênitas estão sumarizadas na Tabela 3 . Observou-se uma parcela significativa de óbitos decorrentes de outras malformações congênitas (Q89) com 272 casos $(51,51 \%)$, seguida por anencefalia (Qoo) com 130 casos $(24,62 \%)$. Também merecem destaque os casos de hidrocefalia e espinha bífida com 32 casos (6,06\%) (Tabela 2). A categoria Q89 da CID-10, 
Capítulo XVII, é representada por malformações congênitas do baço, das suprarrenais e de outras glândulas endócrinas, além de Situsinversus, reunião de gêmeos, malformações múltiplas não classificadas em outra parte (NCOP) e outras malformações especificadas ou não especificadas.

Considerando a análise isolada de cada categoria CID-10 para malformações congênitas, observouse aumento no número absoluto de defeitos congênitos do coração (+100\%) e nos casos de síndromes cromossômicas (+200\%), com destaque para síndrome de Down (n=7) e Síndromes de Edwards e Patau (n=7).

Tabela 3 - Causas específicas de óbitos fetais por malformações congênitas no estado do Maranhão, 2006- 2016

\begin{tabular}{llllllllllllllll}
\hline $\begin{array}{l}\text { Causas do } \\
\text { óbito }\end{array}$ & $\mathbf{2 0 0 6}$ & $\mathbf{2 0 0 7}$ & $\mathbf{2 0 0 8}$ & $\mathbf{2 0 0 9}$ & $\mathbf{2 0 1 0}$ & $\mathbf{2 0 1 1}$ & $\mathbf{2 0 1 2}$ & $\mathbf{2 0 1 3}$ & $\mathbf{2 0 1 4}$ & $\mathbf{2 0 1 5}$ & $\mathbf{2 0 1 6}$ & Total \\
$\begin{array}{l}\text { Hidrocefalia e } \\
\text { espinha bífida }\end{array}$ & 05 & 03 & 03 & 04 & 02 & 05 & 02 & 01 & 02 & 05 & 00 & 32 \\
$\begin{array}{l}\text { Anencefalia } \\
\text { Outras }\end{array}$ & 13 & 10 & 08 & 15 & 13 & 16 & 16 & 11 & 10 & 11 & 07 & 130 \\
$\begin{array}{l}\text { malformações } \\
\text { do sistema } \\
\text { nervoso }\end{array}$ & 03 & 01 & 00 & 02 & 01 & 05 & 01 & 03 & 04 & 04 & 06 & 30 \\
$\begin{array}{l}\text { Malformação } \\
\text { congênita do } \\
\text { coração }\end{array}$ & 02 & 04 & 02 & 02 & 00 & 01 & 04 & 04 & 04 & 04 & 04 & 31 \\
$\begin{array}{l}\text { Outras } \\
\text { malformações } \\
\text { congênitas } \\
\text { do aparelho } \\
\text { circulatório }\end{array}$ & 01 & 00 & 00 & 01 & 00 & 00 & 00 & 00 & 01 & 00 & 01 & 04 \\
$\begin{array}{l}\text { Síndrome de } \\
\text { Down e outras } \\
\text { anomalias } \\
\text { cromossômicas }\end{array}$ & 02 & 00 & 02 & 01 & 01 & 02 & 04 & 01 & 02 & 08 & 06 & 29 \\
$\begin{array}{l}\text { Outras } \\
\text { malformações } \\
\text { congênitas }\end{array}$ & 25 & 17 & 32 & 30 & 12 & 23 & 26 & 26 & 27 & 32 & 22 & 272 \\
\begin{tabular}{l} 
Total \\
\hline
\end{tabular} & $\mathbf{5 1}$ & $\mathbf{3 5}$ & $\mathbf{4 7}$ & $\mathbf{5 5}$ & $\mathbf{2 9}$ & $\mathbf{5 2}$ & $\mathbf{5 3}$ & $\mathbf{4 6}$ & $\mathbf{5 0}$ & $\mathbf{6 4}$ & $\mathbf{4 6}$ & $\mathbf{5 2 8}$ \\
\hline
\end{tabular}

Fonte: Os autores (2018).

\section{Discussão}

Este estudo avaliou a contribuição do vetor malformações congênitas e sua tendência no que diz respeito à mortalidade fetal na série histórica de 2006 a 2016 no estado do Maranhão. Ao longo desse período, observou-se aumento discreto, porém significativo, tanto dos coeficientes de mortalidade fetal geral (7\%), quanto do específico por malformação congênita (5\%), mesmo com a diminuição do número absoluto de óbitos fetais. No mesmo período, observou-se também diminuição do número de nascidos vivos, demonstrando nítida colinearidade dessas estatísticas vitais.

Por se tratar de uma série de onze anos, esperava-se encontrar uma tendência decrescentenos indicadores de mortalidade fetal (geral e específico por MC). Desse modo, é possível inferir que as ações de caráter preventivo dos comitês estadual e municipal de prevenção do óbito infantil e fetal não são suficientes para o controle e a evitabilidade da mortalidade perinatal. Cabe ressaltar que os óbitos fetais por malformações congênitas são, em parte, evitáveis ${ }^{27} \mathrm{e}$, portanto, sua ocorrência denota fragilidade em sua vigilância e negligência do setor 
público no que se refere a melhorar seu desempenho. A baixa visibilidade do óbito fetal é, em alguma medida, explicada por não compor as metas de desenvolvimento do milênio estabelecidas para o ano de $2015^{28}$.

Neste estudo, mais da metade dos óbitos foi classificada como evitável. Mortes evitáveis são consideradas um 'evento sentinela' na avaliação da qualidade da atenção à saúde prestada no pré-natal, no parto e ao recémnascido. Desse modo, a elevada proporção de mortes evitáveis mostra a necessidade de melhoria da assistência pré-natal e ao parto, bem como de políticas de saúde que enfoquem o atendimento integral à mulher e ao parto.

$\mathrm{O}$ ano que apresentou os maiores valores em relação aos indicadores selecionados para o estudo foi exatamente 2015, período correspondente à epidemia provocada pelo vírus zika e à ocorrência da síndrome congênita relacionada a ele. No período de 2015 a 2016, foram confirmados 2.018 casos de microcefalia devido à infecção congênita de recém-nascidos vivos no Brasil. Destes, 140 ocorreram no estado do Maranhão ${ }^{29}$. Neste mesmo estado, no que se refere aos óbitos fetais, neonatais e infantis foram notificados 53 casos suspeitos, seis confirmados, cinco prováveis, 36 descartados, quatro inconclusivos e dois em investigação.

Outro aspecto importante a ser discutido é o aumento e a melhora da notificação dos óbitos fetais no estado do Maranhão após a implantação dos comitês de investigação de óbito infantil e fetal. Um estudo conduzido neste estado, no ano de 2008, mostrou a ocorrência de sub-registro dos óbitos fetais na magnitude de $9,7 \%$ e que ele é maior em municípios menores do estado ${ }^{30}$. Sendo assim, a melhora na qualidade da informação sobre óbitos fetais é fundamental para a construção de indicadores fidedignos e que possam indicar ações mais especificas para enfrentamento deste problema de saúde pública.

O coeficiente de mortalidade fetal geral variou de 12,36/1.0oo nascidos (2008) a 14,65 (2015). Nestes anos, a contribuição da mortalidade por malformações congênitas foi de 2,96\% e 3,72\%, respectivamente. Os valores observados estão abaixo das metas de 12 ou menos óbitos fetais/1.0oo nascimentos estabelecidos pela OMS e pelo Unicef ${ }^{28}$. O óbito fetal é um problema multifatorial, e uma abordagem agregada não permite avaliação de exposição de âmbito individual; entretanto, além de fatores genéticos e/ou ambientais, a baixa qualidade/adesão no pré-natal, dificuldades de diagnóstico precoce e aconselhamento reprodutivo/ genético para interrupção de gestações de risco, bem como diminuição do risco de recorrência, podem estar associados aos números elevados de óbitos fetais.

Apesar da recomendação do uso do coeficiente de mortalidade geral apenas para unidades federativas, nas quais o indicador pode ser calculado pelo método direto, poucos estudos têm sido desenvolvidos em cada uma delas no Brasil, o que dificulta a comparação dos resultados. A literatura revisada mostra que, entre 2001 e 2011, a taxa de mortalidade fetal, no país, caiu de 12,3/1.000 nascimentos para 10,71/1.000 nascimentos, e que os estados da Região Nordeste são os que apresentam as maiores TMF. Observa-se uma tendência à diminuição da mortalidade fetal no território nacional, e que as maiores taxas concentram-se nas regiões Nordeste e Norte e as menores nas regiões Sudeste e Sul ${ }^{4,11}$.

Um estudo realizado, no estado de Pernambuco, sobre os óbitos fetais no período de 2000 a 2011 mostrou uma taxa de mortalidade fetal geral de 11,8 óbitos/1.00o nascimentos. No período estudado, houve diminuição da TMF de 12,1/1.00o nascimentos para 10,2/1.00o nascimentos. Além disso, as malformações congênitas constituíram a segunda causa de morte fetal, com prevalência de $4,8 \%{ }^{31}$.

Neste estudo, como mencionado anteriormente, a porcentagem dos óbitos por MC no Maranhão entre 2006 e 2016 foi de 2,66\%, valor inferior ao encontrado para o estado de Pernambuco no período de 2000 a 2011. Entretanto, quando se comparam as causas de óbito por MC, os resultados são similares, uma vez que os autores observaram maior proporção dos óbitos por malformações congênitas não-especificadas (37,8\%); seguido dos óbitos por anencefalia (26,1\%); e por malformação não-especificadas do coração $(5,9 \%)^{30}$. Cabe salientar que essas comparações não são rigorosas no sentido científico do termo, pois se referem a períodos distintos e a apenas duas unidades federativas; porém, diante do pouco número de estudos, são as únicas possíveis para dar uma ideia do cenário dos óbitos por malformação congênita em parte da Região Nordeste durante os primeiros 16 anos deste século (2000-2016). 
Estudos mostram que os fatores de risco classificados como fetais envolvem o baixo peso fetal ${ }^{32,33} \mathrm{e}$ as malformações congênitas, sendo estas apontadas como a maior causa de óbito quando este se refere ao feto.

Observou-se para o Maranhão que as malformações por "outras malformações" - (Q89 CID-10) representaram mais da metade dos óbitos fetais por MC. Este resultado pode ser considerado negativo, frente à imprecisão do diagnóstico médico. Óbitos sem causa específica, além de prejudicar a construção de indicadores de saúde, dificultam ações de prevenção de recorrência ou redução na taxa de mortalidade fetal.

Pesquisas recentes mostram uma variação entre 14 e $32 \%$ de mortes entre fetos com algum tipo de malformação congênita grave, tais como, as malformações do sistema nervoso central, ou ainda, múltiplas malformações ${ }^{34,35}$. Quando comparado a fetos saudáveis, o risco de mortalidade intrauterina de um feto com MC pode ser cinco vezes maior ${ }^{36}$. Nestes casos, é fundamental a investigação diagnóstica por meio do estudo citogenético para compreender a etiologia da morte fetal e, assim, evitar a possibilidade malformações congênitas futuras, somada à recomendação de aconselhamento genético ${ }^{37}$. Cabe ressaltar que no estado do Maranhão, durante o período estudado, cerca de um quarto dos óbitos por MC ocorreram por defeitos do tubo neural, com destaque para a anencefalia $(24,62 \%)$.

Os defeitos do tubo neural (DTN) seguem um padrão de herança multifatorial com a interação de múltiplos genes bem como de fatores ambientais. Tais defeitos possuem prognóstico ruim e podem ser evitados com suplementação de ácido fólico ( $4 \mathrm{mg} /$ dia pelo menos três meses antes da gestação), possuindo risco de recorrência de $2 \%$ a $4 \%$ para gestações subsequentes ${ }^{38}$.

Alguns casos raros de DTN podem apresentar herança autossômica dominante ou recessiva em diversas gerações de uma família. Nestes casos, podem haver crianças ou fetos com espinha bífida, anencefalia, ou outros subtipos de DTN, e alguns casos de anencefalia podem estar associados a formas não balanceadas de anormalidades cromossômicas estruturais. Nessas famílias, outras malformações e defeitos congênitos podem estar presentes ${ }^{37}$.

Quanto à efetividade da suplementação com ácido fólico, um estudo realizado no estado do Maranhão no período pré e pós-fortificação de farinhas com ácido fólico mostrou que a suplementação não foi capaz de reduzir de forma significativa a incidência de espinha bífida no período estudado ${ }^{39}$. Por outro lado, diversos estudos demonstram que o ácido fólico é um agente preventivo e que reduz o risco de anencefalia e de outros DTN em aproximadamente dois terços ${ }^{40-43}$.

A anencefalia é um defeito grave do desenvolvimento do sistema nervoso central no qual o cérebro e a caixa craniana são malformados. Compõe o espectro dos DTN e resulta da falha de fechamento do tubo neural, no período entre a terceira e a quarta semanas do desenvolvimento, que leva à perda fetal, ou seja, a um natimorto, ou à morte alguns dias após o nascimento, conhecida como morte neonatal ${ }^{44-47}$.

No Brasil, a prevalência estimada de anencefalia é de uma criança para cada mil nascimentos. Cabe destacar que, no país, em 2014, a incidência de óbitos de fetos com anencefalia foi de cerca de 18 casos para cada 10 mil nascidos vivos, uma taxa mais de cinquenta vezes maior que a observada em países como a França, Bélgica ou Áustria. Mais de 50\% dos fetos anencéfalos morrem no útero materno, 49\% sobrevivem menos de 24 horas após o parto e $1 \%$ desses fetos vivem apenas uma semana ${ }^{48,49}$.

Com relação às síndromes cromossômicas, observou-se aumento acentuado no período estudado, representando 5,49\% da casuística. A frequência de cromossomopatias em seres humanos é elevada. Estima-se sua deteç̧ão em 0,5\% dos recém-nascidos, $5 \%$ em óbitos fetais e 50\% em abortos espontâneos ${ }^{50}$. Estes resultados estão de acordo com os achados do estudo aqui apresentado.

A idade materna avançada continua sendo o fator de risco mais provável para a não-disjunção, fenômeno biológico relacionado à ocorrência de aneuploidias, tais como as trissomias do 13, 18 e 21, encontradas neste estudo. Constituem a situação mais comum de distúrbios cromossômicos humanos, ocorrendo em $5 \%$ de todas as gestações conhecidas ${ }^{51}$.

O diagnóstico pré-natal de anomalias cromossômicas é um importante recurso para gestantes com história familiar de cromossomopatia ou com detecção de anomalia fetal na ultrassonografia. O diagnóstico 
etiológico do feto é uma ferramenta essencial para o aconselhamento genético, permitindo estabelecer o prognóstico da gestação em curso, o planejamento do parto, bem como estimar o risco de uma futura gestação, garantindo o apoio necessário para o casal ${ }^{50}$.

A detecção pré-natal de cromossomopatias e de uma série de anormalidades fetais como defeitos cardíacos, hérnia diafragmática, onfalocele, malformações esqueléticas, anomalias renais, uropatia obstrutiva e diversas doenças gênicas, pode ser realizada por translucência nucal (TN) em fetos. Além disso, a triagem combinando a idade materna, a translucência nucal e os marcadores séricos de primeiro trimestre identifica $90 \%$ de gestações com trissomia do $21^{50}$.

Considerando os óbitos por malformações congênitas como causas evitáveis de óbito fetal, o projeto Vigimoma (Vigilância de Morbidade Materna), uma colaboração entre academia e comitês de mortalidade materna municipais e regionais, avaliou e revisou todos os eventos perinatais adversos em Campinas (SP) no período de outubro a dezembro de 2005. Os resultados mostraram uma porcentagem de óbitos fetais por $\mathrm{MC}$ da ordem de $25 \%$. Além disso, os autores realizaram um trabalho visando à evitabilidade potencial, no qual propuseram as principais intervenções possíveis para prevenir os óbitos fetais e neonatais precoces ${ }^{52}$.

Entre as intervenções propostas pelos autores, encontram-se: educação de mulheres grávidas sobre sinais de alerta pré-natais, melhoria na prática clínica pré-natal, promoção do uso de ácido fólico na presença prévia de DTN, qualificação dos registros anatomopatológicos na perinatalogia, promoção da investigação da morte intrauterina e neonatal, promoção de aconselhamento pós-parto com avaliação genética, promoção da importância do cuidado pré-natal e facilitação de sua iniciação precoce, registro de informações sobre o cuidado pré-natal nos registros médicos das pacientes, promoção de aconselhamento pós-parto independentemente da necessidade de avaliação genética e promoção de planejamento familiar ${ }^{2}$.

A limitação do estudo em que se baseia este artigo consiste na utilização de dados secundários, que podem sofrer o efeito da subnotificação. Entretanto, por se tratar de um estudo descritivo e levando-se em conta que as bases de dados utilizadas foram o SIM e o Sinasc, sistemas que possuem boa cobertura, considera-se o viés de informação pequeno. Além disso, a incompletude dos dados é mais nítida para as variáveis sociodemográficas, as quais não foram utilizadas neste estudo de tendências.

\section{Considerações Finais}

Os coeficientes de mortalidade fetal geral e específico por malformações congênitas apresentaram tendência crescente na série 2006-2016 no estado do Maranhão. Mais da metade dos óbitos fetais por malformação congênita foram classificados como evitáveis. Estes achados reforçam que as ações dos comitês de investigação dos óbitos infantis e fetais devem ser mais eficientes para a prevenção e o controle dos óbitos fetais no estado do Maranhão. Em adição, os resultados apontam uma atenção materno-infantil deficitária, uma vez que mesmo com a diminuição da mortalidade infantil no estado, a tendência crescente à mortalidade por malformações congênitas foi demonstrada neste estudo.

\section{Referências}

1. Ministério da Saúde (BR). Secretaria de Vigilância em Saúde. Secretaria de Atenção à Saúde. Manual de vigilância do óbito infantil e fetal e do Comitê de Prevenção do Óbito Infantil e Fetal. 2. ed. Braślia; 2009.

2. Andrade LG, Amorim MMR de, Cunha ASC da, Leite SRR, Vital SA. Fatores associados à natimortalidade em uma maternidade-escola em Pernambuco: estudo caso-controle. Rev Bras Ginecol Obstet. 2009;31(6):285-92.

3. Blencowe H, Cousens S, Jassir FB, Say L, Chou D, Mathers C et al. National, regional, and worldwide estimates of stillbirthrates in 2015, with trends from 2000: a systematic analysis. Lancet Glob Health. 2016;4(2):98-108.

4. Barros PS, Aquino EC, Souza MR. Mortalidade fetal e os desafios para a atenção à saúde da mulher no Brasil. Rev Saude Publica. 2019;53(12):1-10. 
5. Lawn JE, Blencowe H, Pattinson R, Cousens S, Kumar R, Ibiebele I. Stillbirths: Where? When? Why? How to make the data count? Lancet. 2011;377(9775):1448-63.

6. Cecatti JG, Aquino MMA. Causas e fatores associados ao óbito fetal. Rev Ci Med. 1998;7(2):43-8.

7. Lawn J, Shibuya K, Stein C. No cry at birth: global estimates of intrapartum stillbirths and intrapartum-related neonatal deaths. Bull World Health Organ. 2005;83(6):409-17.

8. Fonseca SC, Coutinho ESF. Fatores de risco para mortalidade fetal em uma maternidade do Sistema Único de Saúde, Rio de Janeiro, Brasil: estudo caso-controle. Cad Saúde Pública. 2010;26(2):240-52.

9. Almeida MF, Alencar GP, Novaes HMD, França Junior I, Siqueira AAF, Campbell OM et al. Risk-factors for antepartum fetal deaths in the city of São Paulo, Brazil. Rev Saúde Pública. 2007;41(1):35-43.

10. Barbeiro FMS, Fonseca SC, Tauffer MG, Ferreira MSS, Silva FP, Ventura PM et al. Óbitos fetais no Brasil: revisão sistemática. Rev Saúde Pública. 2015;49(22):1-15.

11. Ministério da Saúde (BR). Portaria MS/GM no 72, de 11 de janeiro de 2010. Estabelece que a vigilância do óbito infantil e fetal é obrigatória nos serviços de saúde (públicos e privados) que integram o SUS. DOU [Internet], 2010 [citado em 2019 nov. 13]; Sec. 1:29-31. Disponível em: http://bvsms.saude.gov.br/bvs/ saudelegis/gm/2010/prt0072 $11012010 . \mathrm{html}$.

12. IPEA. Atlas do Desenvolvimento Humano [Internet]. 2013 [citado em 20 fev 2015]. Disponível em: http:// atlasbrasil.org.br/2013/pt/perfil/.

13. IBGE. Internações hospitalares por doenças relacionadas ao saneamento. Sidra [Internet]. 2013 [citado em 2015 nov 5]. Disponível em: https://sidra.ibge.gov.br/tabela/898.

14. IBGE. Sistema IBGE de Recuperação Automática: Banco de Dados Agregados [Internet].[data desconhecida] [citado em 2018 mar 13]. Disponivel em: http://www.sidra.ibge.gov.br/bda/acervo/ default.asp? $\mathrm{z}=\mathrm{t} \& \mathrm{O}=3 \& \mathrm{i}=$ Phttp.

15. Controladoria Geral da União (BR). Portal da Transparência [Internet]. c2019 [citado em 2018 fev 12]. Disponível em: http://www.portaldatransparencia.gov.br/PortalTransparenciaPesquisaAcaoUF.asp?codigo Acao $=8442 \&$ codigoFuncao $=08 \&$ NomeAcao $=$ Transfer $\% E A n c i a+d e+$ Renda + Diretamente $\% E 0 \mathrm{~s}+$ Fam $\% \mathrm{E}$ Dlias+em+Condi\%E7\%E30+de+Pobreza+e+Extrema+Pobreza+\%28Lei+n\%BA+10 \%2E836\%2C+de+ 2004\%29\&Exercicio $=2016$.

16. Instituto Maranhense de Estudos Socioeconômicos e Cartográficos. Produto Interno Bruto dos Municípios do estado do Maranhão: período 2008 a 2012. São Luís; 2014.

17. Programa das Nações Unidas para o Desenvolvimento, IPEA, Fundação João Pinheiro. Atlas do desenvolvimento humano do Brasil: estado do Maranhão [Internet]. [data desconhecida] [citado em 2018 maio 15]. Disponível em: http://www.atlasbrasil.org.br/2013/pt/perfil uf/maranhao.

18. Secretaria Municipal da Saúde (SP). Coordenação de Epidemiologia e Informação. Declaração de Nascido Vivo: Manual de Anomalias Congênitas. 2 ed. São Paulo; 2012. 97 p.

19. Confidential Enquiry Into Maternal and Child Health. Improving the health of mothers, babies and children [Internet]. London; 2005 [cited 2019 nov 13]. Avaiable from: http://www.cemach.org.uk.

20. Ministério da Saúde (BR). Datasus. Sistema de Informações de Mortalidade: mortalidade por local de residência: Maranhão [Internet]. 2017 [citado em 2018 mar 2]. Disponível em: http://tabnet.datasus.gov. br/cgi/deftohtm.exe?sim/cnv/obt10ma.def.

21. Ministério da Saúde (BR). Datasus. Sistema de Informação de Nascidos Vivos [Internet]. 2017 [citado em 2018 mar 13]. Disponivel em: http://tabnet.datasus.gov.br/cgi/deftohtm.exe?sinasc/cnv/nvMA.def.

22. Morgenstern H. Ecologic studies. In: Rothman KJ; Greenland S, editors. Modern epidemiology. 2nd ed. Philadelphia: Lippincott-Raven Publishers; 1998.

23. Boyle P, Parkin DM. Statistical methods for registries. In: Jensen OM, Parkin DM, Maclennan R, Muir CS, Skeet RG, editors. Cancer Registration: principles and Methods. Lyon: IARC; 1991. p. 126-58. (IARC Scientific Publications, n. 95).

24. Kleinbaum DG, Kupper LL, Muller KE. Applied Regression Analysis and other Multivariable Methods. Belmont, California: PWS-KENT Publishing Company; 1988.

25. Morettin PA, Toloi CM. Séries Temporais. São Paulo: Atual; 1986. (Coleção Métodos Quantitativos). 
26. Ayres M, Ayres Júnior M, Ayres DL, Santos AS. BioEstat 5.0: aplicações estatísticas nas áreas das ciências biológicas e médicas. Belém: MCT; IDSM; CNPq; 2007. 364 p.

27. Malta DC, Sardinha LMV, Moura L, Lansky S, Leal MC, Szwarcwald CL et al. Nota Técnica: Atualização da lista de causas de mortes evitáveis por intervenções do Sistema Único de Saúde do Brasil. Epidemiol Serv Saúde. 2010;19(2):173-6.

28. Victora CG, Requejo JH, Barros AJD, Berman P, Bhutta Z, Boerma T et al. Countdown to 2015: a decade of tracking progress for maternal, newborn, and child survival. Lancet. 2016;387(10032):2049-59.

29. França GVA, Pedi VD, Garcia MHO, Carmo GMI, Leal MB, Garcia LP. Síndrome congênita associada à infecção pelo vírus Zika em nascidos vivos no Brasil: descrição da distribuição dos casos notificados e confirmados em 2015-2016. Epidemiol. Serv. Saude. 2018;27(2):1-12.

30. Rafael RAA, Ribeiro VS, Cavalcante MCV, Santos AM, Simões VMF. Relacionamento probabilístico: recuperação de informações de óbitos infantis e natimortos em localidade do Maranhão, Brasil. Cad Saude Publica. 2011;27(7):1371-9.

31. Holanda AS. Caracterização da mortalidade fetal em Pernambuco,de 2000 a 2011: causas e fatores associados [monografia]. Recife: Fundação Oswaldo Cruz; 2013.

32. Smith GC, Shah I, White IR, Pell JP, Dobbie R. Previous preeclampsia, preterm delivery, and delivery of a small for gestational age infant and the risk of unexplained stillbirth in the second pregnancy: a retrospective cohort study, Scotland, 1992-2001. Am J Epidemiol. 2007;165(2):194-202.

33. Yildirim G, Aşicioğlu O, Güngördük K, Turan I, Acar D, Aslan H et al. Subsequent obstetrics outcomes after intrauterine death during the first pregnancy. J Matern Fetal Neonatal Med.2014;27(10):1029-32.

34. Vieira MSM, Siebert EC, Ceglio WQGW, Almeira MH, Batista TS, Freitas PF. Dificuldades para a identificação da causa do óbito fetal: como resolver? Rev Bras Ginecol Obstet. 2012;34(9):403-8.

35. Barros ML, Fernandes DA, Melo EV, Porto RLS, Maia MCA, Godinho AS et al. Malformações do sistema nervoso central e malformações associadas diagnosticadas pela ultrassonografia obstétrica. Radiol Bras. 2012;45(6):309-14.

36. Neto NC, Souza ASR, Moraes Filho OB, Noronha AMB. Volume do líquido amniótico associado às anomalias fetais diagnosticadas em um centro de referência do nordeste brasileiro. Rev Bras Ginecol Obstet. 2009;31(4):164-70.

37. Pereira TM, Oliveira ARCP, Teixeira ACZ, Jesus NA, Rodrigues G, Agostinho MAB et al. Frequência das anormalidades cromossômicas: importância para o diagnóstico citogenético. Arq Ciênc Saúde. 2009;16(1):31-3.

38. Tinker SC, Devine O, Mai C, Hamner HC, Reefhuis J, Gilboa SM et al. Estimate of the potential impact of folic acid fortification of corn masa flour on the prevention of neural tube defects. Birth Defects Res A Clin Mol Teratol. 2013;97(10):649-57.

39. Silva BFS, Pires RCR, Silva VC. Incidência de espinha bífida no estado do Maranhão, pré- e pós-fortificação de farinhas com ácido fólico e fatores maternos associados. Rev Mar Saud Col. 2016;1(3):133-140.

40. Oakley GPJ. The scientific basis for eliminating folic acid-preventable spina bifida: a modern miracle from epidemiology. Ann Epidemiol. 2009;19(4):226-30.

41. Berry RJ, Li Z, Erickson JD, Li S, Moore CA, Wang H et al. Prevention of neural-tube defects with folic acid in China. China-U.S. Collaborative Project for Neural Tube Defect Prevention. N Engl J Med.1999;341(20):1485-90.

42. Brent RL, Oakley GP, Md J. The unnecessary epidemic of folic acid-preventable spina bifida and anencephaly. Pediatrics. 2000;106(4):825-7.

43. Wilson RD, Audibert F, Brock JA. Pre-conception Folic Acid and Multivitamin Supplementation for the Primary and Secondary Prevention of Neural Tube Defects and Other Folic Acid-Sensitive Congenital Anomalies. J Obstet Gynaecol Can. 2015;37(6):534-52.

44. Botto LD, Moore CA, Khoury MJ, Erickson JD. Neural-tube defects. N Engl J Med. 1999; 341(20):1509-19.

45. Campbell LR, Dayton DH, Sohal GS. Neural tube defects: a review of human and animal studies on the etiology of neural tube defects. Teratology. 1986;34(2):171-87.

46. Russell SA, McHugo JM, Pilling D. Cranial abnormalities. In: Twining P, McHugo JM, Piling D., editors. Textbook of Fetal Anomalies. 2nd ed. Churchill Livingstone: Elsevier; 2007. p. 95-141. 
47. Obeidi N, Russell N, Higgins JR, O'Donoghue K. The natural history of anencephaly. Prenat Diagn. 2010;30(4):357-60.

48. Campos APM. Anencefalia [Internet]. 2012 [citado em 2018 jan 18]. Disponível em: http://enfermagemamoreiracampos2011.blogspot.com/2012/04/anencefalia.html.

49. Jaquier M. Relatório sobre nascimento e a vida de bebês com anencefalia [Internet]. Anencephaly. Info. Brasil, 2012 [citado em 2018 jan 18]. Disponível em: http://www.anencephaly.info/p/relatorio.php.

50. Gus R. Diagnóstico pré-natal das anomalias citogenéticas. In: Maluf SW, organizador. Citogenética humana. Porto Alegre: Artmed; 2011. p. 280-85.

51. Fraga DD. Alterações cromossômicas numéricas. In: Maluf SW, organizador. Citogenética humana. Porto Alegre: Artmed; 2011. p. 70-9.

52. Amaral E, Souza JP, Surita F, Sousa MH, Cecatti JG, Campbell O. A population-based surveillance study on severe acute maternal morbidity (near-miss) and adverse perinatal outcomes in Campinas, Brazil: The Vigimoma Project. BMC Pregnancy and Childbirth; 2011;11(9):1-8. 